\title{
A Innovative Prognostic Symbol Based on Autophagy-Related Long Noncoding RNAs Signature Predicts in Low-Grade Glioma
}

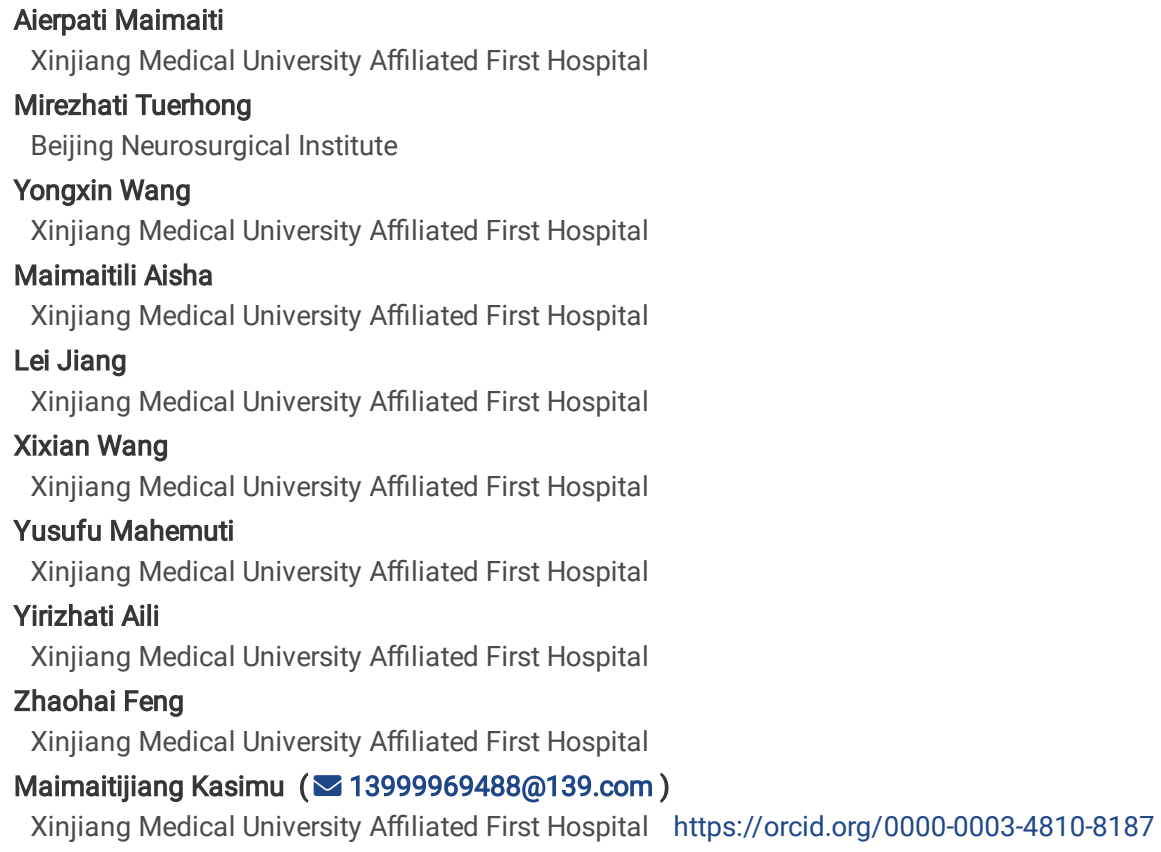




\section{Abstract}

Autophagy is a highly conserved lysosomal degradation process essential in tumorigenesis. However, the involvement of autophagy-related IncRNA in lowgrade gliomas (LGG) remains a pending question. Efforts were made to establish an autophagy-related IncRNA signature prognostic in LGG patients, and to explore the behind potential function. We used Univariate Cox, Least Absolute Shrinkage and Selection Operator (Lasso), and Multivariate Cox regression models were designed to establish an autophagy-related IncRNA prognostic signature. Kaplan-Meier survival analysis, receiver operating characteristic (ROC) curve, nomogram, C-index, calibration curve and clinical decision-making curve were adopted to assess the predictive capability of the identified signature.A signature comprising 9 autophagy-related IncRNAs (AL136964.1, ARHGEF26-AS1, PCED1B-AS1, AS104072.1, PRKCQ-AS1, LINC00957, AS125616.1, PSMB8AS1, and AC087741.1) was identified as a prognostic model. LGG patients were allocated into high- and low-risk cohorts depending on the median modelbased Riskscore. The survival analysis showed a 10-year survival rate of 9.3\% (95\% Cl: 1.91-45.3\%) in high-risk LGG patients and 48.4\% (95\% Cl: $24.7-95.0 \%)$ in low-risk individuals in the training set. While those of patients in the validation set were $13.48 \%$ (95\% Cl: $4.52-40.2 \%)$ for high-risk LGG patients and $48.4 \%$ (95\% Cl: 28.04-83.4\%) for low-risk LGG patients, respectively. This suggested a relatively low survival in high-risk cases compared to low-risk individuals. In addition, LncRNA signature was independently prognostic and potentially associated with the progression of LGG. Taken together, our constructed 9 autophagy-related IncRNA signature may play a crucial part in the diagnosis and treatment for LGG, which may guide to open up a new avenues for tumor targeted therapy.

\section{Introduction}

Low-grade glioma (LGG) is one of the most prevailing and invasive malignancies in adult brain, and the incidence is increasing globally[1]. It is reported that the median survival for LGG patients is 5 to 10 years, compared with 14 months for those with glioblastoma[2], which allows patients to survive for up to 20 years[3]. However, around 50-75\% of LGG patients have a disease progressed generally to a high-grade level, leading to neurological disability and death[4, 5]. According to the statistics of Central Brain Tumor Registry of the United States (CBTRUS), approximate 70\% of primary malignant brain tumors are diagnosed as gliomas[6], and more than 14,000 new cases occur every year with the annual incidence rate of about 5/100,000, of which LGG accounts for more than $15 \%[7]$. Although maximum surgical resection, radiotherapy and chemotherapy are considered as the main strategies combating LGG, tumor metastasis, recurrence and patient death cannot be completely prevented[8]. Therefore, there is a need to further explore additional tools for the diagnosis treatment and prognosis of LGG and to develop new risk factors and molecular markers for early stratification of low- and high-risk patients with preventive treatment[9]. In this context, identifying high-risk LGG individuals with unsatisfying outcome turns out an essential work that needs to be concerned. Clinicopathological characteristics, such as type, age, extent of resection (postoperative MRI), tumor size, KPS score, tumor grade, chromosome 1p/19q codeletion and IDH1/2 mutation status, can be factors involved in prognosis of LGG[10]. Recently, some molecular characteristics have been proposed as predictors of LGG outcome, such as models associated with immunity[11], DNA methylation[12], m6A-related gene[13], and alternative splicing event[14]. Nevertheless, the involvement of autophagy-related long non-coding RNA (IncRNA) in LGG prognosis is unclear.

Autophagy is a series of structural evolution processes of autophagosomes that are finely regulated by autophagy-related genes (ATG)[15]. Under the action of drugs, starvation, hypoxia or other factors, a double-layer partitive membrane can be seen around a cell component to be degraded, and then gradually extends to make the cytoplasmic component to be degraded completely sealed, the product of which is called autophagosome[16, 17]. Assisted by cytoskeleton microtubule system, the autophagosome formed is delivered to a lysosome, by which the two are fused and an autolysosome is correspondingly generated. The content inside the autolysosome is degraded and utilized by cells under the action of lysosomal enzymes[18]. It is reported that autophagy involves a variety of signaling pathways, while mainly fall into AMPK and mTOR signaling pathways[19], with the former being a promoter of autophagy and the latter reversely a suppressor[20]. In addition, there are many classical apoptotic signaling pathways or proteins reported having complex associations with autophagy regulation[21]. For example, p53 induces autophagy with mTOR suppression through transcriptional activation of AMPK and TSC1/TSC2[22, 23]. Intranuclear p53 can transcriptionally activate damage regulated autophagy modulator (DRAM) to improve autophagy[24]. Cytoplasmic p53 mediates autophagy in a complex combining HMGB1, by which targeted knock out of cytoplasmic p53 gene can improve HMGB1 expression to induce autophagy, while knock out of HMGB1 can reversely improve cytoplasmic p53 content leading to autophagy suppression[25]. Besides, the involvement of integrin-mediated signaling pathways in the mechanism behind tumor metastasis by autophagy is also identified[26].

LncRNAs are a group of RNA molecules incapable of encoding proteins and characterized by 200 to 100,000 nt in length[27]. In addition to modulating gene expression at the epigenetic, transcriptional and post-transcriptional levels, they can also intervene in processes such as $\mathrm{X}$ chromosome silencing, chromosome and genomic modifications, transcriptional interference, transcriptional activation, and nuclear translocation.[28]. Increasing scholars have paid more attention to the role of IncRNAs in gliomas, which has been proven in the dynamic process of autophagy and even the progress of most tumors via modulating ATGs in transcriptional or post-transcriptional level. Recent evidence suggests that IncRNA H19 mediates the proliferative activity and autophagy of glioma cells through the mTOR/ULK1 pathway, where H19 over expression suppresses autophagy of U87 cells while H19 silence makes the autophagy enhanced[29]. Knocking down IncRNA DLEU1 inhibits glioma progression and promotes chemosensitivity of temozolomide by regulating autophagy[30]. LINCRA1 stabilizes H2B K120 monoubiquitination (H2Bub1) by binding H2B and impeding H2Bub1 and ubiquitin-specific protease 44 (USP44) interaction, resulting in autophagy suppression and development of radio-resistance of gliomas[31]. LncRNA MALAT1 knockout leads to the inhibition of cell migratory and invasive abilities with the autophagy of miR-384/GOLM1 axis in glioma blocked[32]. Additionally, LINC00470 mediates the epigenetic regulation of ELFN2 to disperse autophagy of glioblastoma cells[33]. In this context, there exists an association between IncRNAs and autophagy, which may be involved in the pathogenesis of LGG.

In the research, an autophagy-related signature composed of 9 prognostic IncRNAs was identified to potentially assist in predicting the survival outcome of LGG patients, which may facilitate to customize individualized targeted therapy. Besides, our constructed signature was also proven to be prognostic via a series of regression models. By our research, a new view on prognostic biomarkers and a deep insights into autophagy-related IncRNA in LGG may be offered. 


\section{Materials And Methods}

\subsection{Data acquisition and processing}

RNAseq data along with matched clinical information for LGG tissue samples were extracted from The Cancer Genome Atlas (TCGA) database (https://cancergenome.nih.gov/), including RPKM data from 529 LGG samples. Annotations and classification of IncRNAs and protein-coding genes were completed on the Ensembl human genome Browser GRCh38.p13 (http://asia.ensembl.org/index.html). Normalization of gene expression was down by limma package. Samples having a survival time no more than 30 days or with incomplete clinical data were excluded to eliminate non-cancer related deaths. Finally, 476 samples of LGG were included and grouped at random into training set $(n=240)$ and a validation set $(n=236)$. The training set was taken to construct a prognostic signature, while the validation set and TCGA set $(n=476)$ worked for validation. Two independent validation sets were collected for external validation, including the GSE16011 microarray from Gene Expression Omnibus (GEO) analyzed on the GPL8542 platform Affymetrix GeneChip Human Genome U133 Plus 2.0 Array [CDF: Hs133P_Hs_ENTREZG.cdf] (https://www.ncbi.nlm.nih.gov/geo/query/acc.cgi?acc=GSE16011) and the mRNA-seq-693 dataset (http://www.cgga.org.cn/download.jsp) from Chinese Glioma Genome Atlas (CGGA). Approval by the Ethics Committee or written informed consent of the patient is waived since our data were all from public databases. Clinical characteristics of including LGG patients are depicted in Table 1. 
Table 1

Clinical information for three LGG patients sets in this study.

\begin{tabular}{|c|c|c|c|c|c|}
\hline Covariates & & $\begin{array}{l}\text { training cohort }(n= \\
240)\end{array}$ & $\begin{array}{l}\text { internal validation cohort }(n= \\
236)\end{array}$ & $\begin{array}{l}\text { TCGA cohort } \\
(n=476)\end{array}$ & P-value \\
\hline \multirow[t]{2}{*}{ Age } & $<40$ years & $114(47.5 \%)$ & $106(44.92 \%)$ & $220(46.22 \%)$ & $0.6358^{a}$ \\
\hline & $>=40$ years & $126(52.5 \%)$ & $130(55.08 \%)$ & $256(53.78 \%)$ & \\
\hline \multirow[t]{2}{*}{ Gender } & female & $101(42.08 \%)$ & $115(48.73 \%)$ & $216(45.38 \%)$ & $0.1726^{a}$ \\
\hline & male & $139(57.92 \%)$ & $121(51.27 \%)$ & $260(54.62 \%)$ & \\
\hline \multirow[t]{2}{*}{ Grade } & $\mathrm{G} 2$ & $118(49.17 \%)$ & $113(47.88 \%)$ & $231(48.53 \%)$ & $0.8502^{a}$ \\
\hline & G3 & $122(50.83 \%)$ & $123(52.12 \%)$ & $245(51.47 \%)$ & \\
\hline \multirow[t]{5}{*}{ Diagnoses } & Astrocytoma, anaplastic & $60(25 \%)$ & $61(25.85 \%)$ & $121(25.42 \%)$ & $0.8495^{b}$ \\
\hline & Astrocytoma, NOS & $32(13.33 \%)$ & $26(11.02 \%)$ & $58(12.18 \%)$ & \\
\hline & Mixed glioma & $66(27.5 \%)$ & $59(25 \%)$ & $125(26.26 \%)$ & \\
\hline & $\begin{array}{l}\text { Oligodendroglioma, } \\
\text { anaplastic }\end{array}$ & $34(14.17 \%)$ & $38(16.1 \%)$ & $72(15.13 \%)$ & \\
\hline & Oligodendroglioma, NOS & $48(20 \%)$ & $52(22.03 \%)$ & $100(21.01 \%)$ & \\
\hline \multirow[t]{7}{*}{ First presenting symptom } & Headaches & $50(20.83 \%)$ & $43(18.22 \%)$ & $93(19.54 \%)$ & $0.3481^{b}$ \\
\hline & Mental Status Changes & $24(10 \%)$ & $14(5.93 \%)$ & $38(7.98 \%)$ & \\
\hline & Motor/Movement Changes & $17(7.08 \%)$ & $18(7.63 \%)$ & $35(7.35 \%)$ & \\
\hline & Sensory Changes & $9(3.75 \%)$ & $7(2.97 \%)$ & $16(3.36 \%)$ & \\
\hline & Visual Changes & $3(1.25 \%)$ & $8(3.39 \%)$ & $11(2.31 \%)$ & \\
\hline & Seizures & $114(47.5 \%)$ & $118(50 \%)$ & $232(48.74 \%)$ & \\
\hline & Unknown & $23(9.58 \%)$ & $28(11.86 \%)$ & $51(10.71 \%)$ & \\
\hline \multirow[t]{5}{*}{$\begin{array}{l}\text { First presenting symptom longest } \\
\text { duration }\end{array}$} & $0-30$ Days & $106(44.17 \%)$ & $96(40.68 \%)$ & $202(42.44 \%)$ & $0.6829^{b}$ \\
\hline & 31-90 Days & $33(13.75 \%)$ & $39(16.53 \%)$ & $72(15.13 \%)$ & \\
\hline & 91-180 Days & $16(6.67 \%)$ & $18(7.63 \%)$ & $34(7.14 \%)$ & \\
\hline & $>181$ Days & $55(22.92 \%)$ & $47(19.92 \%)$ & $102(21.43 \%)$ & \\
\hline & Unknown & $30(12.5 \%)$ & $36(15.25 \%)$ & $66(13.87 \%)$ & \\
\hline \multirow[t]{3}{*}{ Seizure history } & YES & $140(58.33 \%)$ & $142(60.17 \%)$ & $282(59.24 \%)$ & $0.824^{\mathrm{a}}$ \\
\hline & NO & $84(35 \%)$ & $80(33.9 \%)$ & $164(34.45 \%)$ & \\
\hline & Unknown & $16(6.67 \%)$ & $14(5.93 \%)$ & $30(6.3 \%)$ & \\
\hline \multirow[t]{2}{*}{ Sample type } & Primary Tumor & $234(97.5 \%)$ & $226(95.76 \%)$ & $460(96.64 \%)$ & $0.4254^{a}$ \\
\hline & Recurrent Tumor & $6(2.5 \%)$ & $10(4.24 \%)$ & $16(3.36 \%)$ & \\
\hline \multirow[t]{3}{*}{ radiation_therapy } & YES & $141(58.75 \%)$ & $130(55.08 \%)$ & $271(56.93 \%)$ & $0.3466^{a}$ \\
\hline & NO & $73(30.42 \%)$ & $83(35.17 \%)$ & 156(32.77\%) & \\
\hline & Unknown & $26(10.83 \%)$ & $23(9.75 \%)$ & $49(10.29 \%)$ & \\
\hline \multirow[t]{3}{*}{ IDH1 mutation status } & Mutant & $42(17.5 \%)$ & $48(20.34 \%)$ & $90(18.91 \%)$ & $0.7295^{a}$ \\
\hline & Wildtype & $14(5.83 \%)$ & $20(8.47 \%)$ & $34(7.14 \%)$ & \\
\hline & Unknown & $184(76.67 \%)$ & 168(71.19\%) & $352(73.95 \%)$ & \\
\hline${ }^{\mathrm{a}}$ Chi square test & & & & & \\
\hline
\end{tabular}

2.2. Screening for autophagy-related IncRNAs 
Human Autophagy Database (HADb; http://www.autophagy.lu/index.html) was consulted to obtain 232 ATGs, the Pearson correlation coefficient of which with the expression of IncRNAs was calculated to give autophagy-related IncRNAs. LncRNAs of an absolute correlation coefficient $>0.7(|R|>0.7)$ and $p<$ 0.001 were selected.

\subsection{Establishment of a prognostic autophagy-related IncRNA signature}

Univariate Cox regression analysis was done to filter IncRNAs associated with overall survival (OS) of LGG patients $(P<0.05)$. The least absolute shrinkage and selection operator (Lasso) penalty-based logistic model involving the IncRNAs filtered in univariate analysis was established for data dimensionality reduction, with 1000 cross-validation performed to prevent over-fitting, and candidate prognostic IncRNAs were identified. Finally, a series of multivariate Cox regression models were constructed, which showed several sets of IncRNAs associated with autophagy as a prognostic signature involving a linear combination of IncRNA expression level and the corresponding regression coefficient. The optimal gene set was identified using the Akaike information criterion (AIC). Riskscore based on the final signature was calculated following the formula as:

ARlncRNA Riskscore $=\sum_{i=1}^{n} \operatorname{coef} A R \ln c R N A_{i} \times \operatorname{Exp}_{\text {ARlncRNA }}$

where ARIncRNA Riskscore is the prognostic Riskscore of patients with LGG, coef is the multivariate Cox regression coefficient, ARIncRNAi is the i-th autophagy-related IncRNA and Exp ARIncRNAi is the corresponding expression level.

Univariate and multivariate analyses were further taken to evaluate whether the identified IncRNA signature was prognostic for LGG independently of clinicopathological characteristics involving age, gender, first_presenting_symptom, first_presenting_symptom_longest_duration, grade, radiation_therapy, seizure_history, diagnoses, type, and IDH1_mutation_status.

\subsection{Evaluation of the IncRNA-based prognostic signature}

LGG samples from the training set were assigned a Riskscore based on which two cohorts were generated as high-risk and low-risk. Kaplan-Meier (KM) curves were plotted to show the OS of the two populations, along with bilateral log-rank sum test run to calculate hazard ratio (HRs) and 95\% confidence interval (Cls) to analyze whether the between-group difference is statistically significant. Receiver operating characteristic (ROC) curves were drawn to evaluate the sensitivity and specificity of the prognostic signature. Finally, further validation was done in the validation sets.

\subsection{Construction and validation of nomogram}

With clinicopathological variables, such as age, gender, diagnoses, grade, radiation_therapy, seizure_history, type, IDH1_mutation_status, and the signaturebased Riskscore both considered, a nomogram was constructed in the training set to predict OS at 1 year, 3 years, and 5 years, with concordance index (Cindex) and corresponding $95 \% \mathrm{Cl}$ calculated to assess the distinguishing and predictive ability. The C-index ranged between $0.5-1.0$, and the higher $\mathrm{C}$-index represents the stronger distinguishing ability of the predictive model. In addition, calibration curves and decision curve analysis (DCA) of the nomogram were generated to identify the consistency between the bias-corrected predicted survival rate and the actual observed survival rate. Finally, the above results were verified in the validation sets.

\subsection{Relationship of autophagy-related IncRNAs with immune infiltrates}

ESTIMATE and CIBERSORT deconvolution algorithms were applied to analyze immune infiltration and stromal score in the training set, to assess and calculate the abundance of 22 immune infiltrates in LGG, and finally to investigate the relationship between immune cell infiltration and the signature-based Riskscore. The above results was further validated in a validation set.

\subsection{Validation of the autophagy-related IncRNA model}

CGGA mRNAseq-693 and GEO GSE16011 data were extracted to validate the prognostic performance for the IncRNA signature. The Riskscore of samples was obtained in the same formula, and the gene expression in patients under different age, grade, IDH mutation status, 1p19q codeletion status, radiation_status, chemotherapy, tumor type (primary and recurrent) and MGMTp codeletion status was compared by boxplots. KM curves for each signature gene were plotted to display survival difference in groups divided by median expression.

\subsection{Gene set enrichment analysis (GSEA)}

Gene Set Enrichment Analysis (GSEA) is a calculation method used to determine whether a pre-defined gene set can show significant consistency differences in two biological states. The predefined gene set is a gene set, which can contain genes of interest, such as a certain pathway, a certain GO term, or a hall marker gene set. Two biological states: a high risk group and a low risk group. It is different from conventional functional enrichment analysis. Routine enrichment analysis must first perform differential screening, and use the screened genes for functional enrichment. This method may miss some key information due to unreasonable screening parameters. And GSEA does not need to do a different analysis, just take the expression of all genes to find the consistent and different pathways between the high risk group and the low risk group. Here, we selected the 'c5.all.v7.2.symbols.gmt' and 'c2.cp.kegg.v7.2.symbols.gmt' from MSigDB database to perform GSEA enrichment analysis. The biological pathways with $\mid$ NES| $>1$, NOM p-val $<0.05$ and FDR q-val $<0.25$ were selected.

\subsection{Statistics}

Statistical analyses were performed by R software (version 4.0.3, https://www.r-project.org/). Perl programming language (version 5.32.1, http://www.perl.org) was used. A difference of $\mathrm{P}<0.05$ was considered statistically significant. 


\section{Results}

\subsection{Screening for autophagy-related IncRNAs of prognostic value}

RNA-seq data of LGG patients in TCGA database screened 13,868 IncRNAs, HADb analysis obtained 232 ATGs, and Pearson analysis identified 80 autophagyrelated IncRNAs $(|R|>0.7, P<0.001)$. Univariate Cox regression analysis obtained 14 out of the 80 IncRNAs closely associated with the OS of LGG cases in the training set. Subsequently, 14 IncRNAs were processed on a Lasso regression model to obtain 13 candidate autophagy-related IncRNAs (Fig. 1A), with the optimal penalty parameter determined through 1000 rounds of cross-validation (Fig. 1B). A stepwise multivariate Cox regression analysis was then done to finally give a 9-IncRNA signature as a prognostic factor for LGG cases (Table 2), comprising IncRNAs AL136964.1, ARHGEF26-AS1, PCED1B-AS1, AC104072.1, PRKCQ-AS1, LINC00957, AC125616.1, PSMB8-AS1, and AC087741.1. A Riskscore indicting LGG prognosis was then established based on the signature using the coefficients in multivariate Cox analysis and the expression levels of 9 signature IncRNAs. The formula is as follows: ARIncRNA Riskscore $=$ $[$ AL136964.1*-1.630] + [ARHGEF26-AS1*-0.329] + [PCED1B-AS1*-0.794] + [AC104072.1*0.142] + [PRKCQ-AS1*-0.488] + [LINC00957*0.457] + [AC125616.1*-1.143] + [PSMB8-AS1*0.457] + [AC087741.1*-0.705]. In addition, a Sankey map was generated to visualize the associations between autophagy-related genes, autophagy-related IncRNAs, and IncRNA risk types (Supplementary Fig. 1).

Table 2

Multivariate Cox regression analyses of 9-autophagy related IncRNAs in Lower-grade glioma with OS.

\begin{tabular}{|llllll|}
\hline ARIncRNAs & coef & HR & HR.95L & HR.95H & pvalue \\
\hline AL136964.1 & -1.630 & 0.196 & 0.095 & 0.406 & $<0.001$ \\
\hline ARHGEF26-AS1 & -0.329 & 0.720 & 0.566 & 0.915 & 0.007 \\
\hline PCED1B-AS1 & -0.794 & 0.452 & 0.325 & 0.630 & $<0.001$ \\
\hline AC104072.1 & 0.142 & 1.153 & 0.994 & 1.336 & 0.059 \\
\hline PRKCQ-AS1 & -0.488 & 0.614 & 0.391 & 0.962 & 0.033 \\
\hline LINC00957 & 0.457 & 1.579 & 1.128 & 2.211 & 0.008 \\
\hline AC125616.1 & -1.143 & 0.319 & 0.170 & 0.597 & $<0.001$ \\
\hline PSMB8-AS1 & 0.457 & 1.580 & 1.208 & 2.066 & $<0.001$ \\
\hline AC087741.1 & -0.705 & 0.494 & 0.259 & 0.942 & 0.032 \\
\hline
\end{tabular}

LGG samples in the training cohort were scored and defined as high-risk when the score was greater than the median sore and as low-risk when a lower score was shown. KM curves revealed a significantly poorer OS in high-risk individuals, relative to low-risk cases $(\mathrm{P}<0.001)$ (Fig. 1C), indicating that the Riskscore was prognostic. Besides, the survival rates at 3,5 and 10 years of the high-risk population were about $59.7 \%, 42.3 \%$ and $9.3 \%$, respectively ( $95 \%$ Cl: $49.6 \%$ $-71.9 \%, 30.45 \%-58.7 \%$ and $1.91 \%-45.3 \%)$, and those of low-risk patients were about $94.4 \%, 75.4 \%$ and $48.4 \%$, respectively $(95 \%$ Cl: $88.4 \%-100 \%, 61.5 \%-92.3 \%$ and $24.7 \%-95.0 \%$ ). Time-dependent ROC curves for OS to 1, 2 and 3 years showed AUC of 0.926, 0.898 and 0.903, respectively (Fig. 1D). Then, Riskscore distribution map, survival scatter plot and expression heatmap were plotted in the training set, which revealed a consistency between the death of LGG patients and the signature-based Riskscore (Fig. 1E). Finally, we also compared the expression of VMP1 gene (a newly discovered autophagy-related gene) in the two risk cohorts, and a significantly lower expression was seen in the low-risk cohort $(P<0.001)$ (Fig. 1F).

\subsection{Validation of the IncRNA signature in the validation set and TCGA set}

We then validated the prognostic power of the IncRNA signature in a validation set of 236 LGG patients and calculated Riskscores which assigned patients into high-risk $(n=114)$ and low-risk $(n=122)$ groups. Similar to the training set, KM analysis demonstrated that the OS of high-risk cases was evidently lower, compared to low-risk cases ( $\mathrm{P}<0.001$ ) (Fig. 2A). The 3-year, 5-year and 10-year OS rates of high-risk patients were about 54.52\%, 37.93\% and 13.48\% respectively (95\% Cl: $43.62 \%-68.1 \%, 26.13 \%-55.0 \%$ and $4.52 \%-40.2 \%)$, while those of low-risk patients were about $92.8 \%, 79.0 \%$ and $48.4 \%$, respectively (95\% Cl: $86.54 \%-99.6 \%, 66.09 \%-94.4 \%$ and $28.04 \%-83.4 \%$ ). ROC curves showed AUC values in 1-year, 2-year and 3-year OS separately of 0.832, 0.860 and 0.879 (Fig. 2B). Riskscore distribution plot, patient survival status and time, and expression heatmap of the high- and low-risk populations in the validation set were revealed in Fig. 2C. VMP1 gene expression was also noted to increase in high-risk cases higher than that of low-risk individuals $(\mathrm{P}<0.001)($ Fig. $2 \mathrm{D})$. To identify the robustness of the IncRNA signature, the total TCGA set was taken for validation with the same classification method. As analyzed, the OS of highrisk patients was poorer relative to that of low-risk patients $(P<0.001)$ (Fig. $2 E$ ), and the AUC values at 1,2 and 3 years of 0 s separately were $0.876,0.876$ and 0.894 (Fig. 2F). The Riskscore distribution plot, patient survival status and time, and expression heatmap in the TCGA set, as well as VMP1 expression ( $<$ < 0.001) (Fig. 2H), showed consistent results with the training and validation sets (Fig. 2G). Overall, the Riskscore based on 9-IncRNA signature could significantly help predict the survival of LGG individuals, whether in the training or the validation and the TCGA sets.

\subsection{Verification of the 9 signature genes and construction of a nomogram}

To identify whether 9 autophagy-related signature IncRNAs is prognostic, KM curves were established in the training set. LINC00957, PCED1B-AS1, and PSMB8-AS1 were negatively correlated with OS, suggesting their potential role as a risk factor since their high expression indicates an adverse outcome. 
AC087741.1, AC104072.1, AC125616.1, AL136964.1, ARHGEF26-AS1, and PRKCQ-AS1 were seen positively correlated with OS (P< 0.001), indicating that they may be protective against the development of LGG (Fig. 3A). Furthermore, a nomogram was constructed in the training set that included age, gender, diagnoses, grade, radiation_therapy, seizure_history, type, IDH1_mutation_status, and the IncRNA signature-based Riskscore (Fig. 3B). The nomogram functioned with each variable assigned a score by multivariate Cox analysis, and then an estimated survival rates at 1,3 , and 5 years were calculated by drawing a vertical line between the total points axis and each prognosis axis. The C-index showed a value of 0.834 (95\% $\mathrm{Cl}$ : $0.780-0.888$ ). The calibration curves (Fig. 3C) and the DCA curves (Fig. 3D) showed that the nomogram had a good predictive performance. Consistent findings were seen in the validation set (Fig. 4A-D). We also made proportional hazards (PH) assumption in the nomogram model (Supplementary Fig. 2).

\subsection{Association of the IncRNA signature with immune infiltrates}

TIMER database was consulted to investigate the relationship between the IncRNA signature and immune infiltrates in LGG. As reflected in Fig. 5A, correlation coefficients of the signature with T cells CD4 memory resting, Macrophages M1 and Macrophages M0 were $0.25,0.32$ and 0.27 , respectively ( $<<0.05)$, which indicated a positive correlation. A negative association of Mast cells activated and Monocytes with the signature-based Riskscore was identified ( $P<0.05)$, suggesting that the IncRNA signature was relevant to the high infiltration of immune cell subtypes. In addition, consistent results were observed in the validation set (Fig. 5B). We also used the single-sample gene-set enrichment analysis (sSGSEA) to quantify the abundance of 28 immune cell infiltration based on the gene set Charoentong's study. [34]The results also indicated the autophagy-related IncRNA signature did not present a significant correlation with immune infiltration (Fig. 5C-D).

\subsection{Verification of the IncRNA model in external datasets}

To identify whether the IncRNA signature is independently prognostic in LGG, univariate and multivariate Cox analyses that included age, gender, first_presenting_symptom, first_presenting_symptom_longest_duration, grade, radiation_therapy, seizure_history, diagnoses, type, IDH1_mutation_status, and the signature-based Riskscore. Multivariate analysis showed that the Riskscore was of significant prognostic value in the training, validation and TCGA sets $(P<0.05)$, while age as a clinical factor also showed a significant association with survival of LGG patients $(P<0.05)(F i g .6 A-F \&$ Table 3$)$. 
Table 3

Univariate and Multivariate Cox regression analysis of the Autophagy-related IncRNAs Riskscore in LGG patient cohorts.

\begin{tabular}{|c|c|c|c|c|c|c|c|}
\hline \multirow[t]{2}{*}{ Variables } & & \multicolumn{3}{|c|}{ Univariable model } & \multicolumn{3}{|c|}{ Multivariable model } \\
\hline & & $\mathrm{HR}$ & $95 \% \mathrm{Cl}$ & P-value & $\mathrm{HR}$ & $95 \% \mathrm{Cl}$ & P-value \\
\hline \multicolumn{8}{|l|}{ Training cohort $(n=240)$} \\
\hline Age & & 1.068 & $1.046-1.089$ & $<0.001$ & 1.072 & $1.046-1.099$ & $<0.001$ \\
\hline Gender & Female/Male & 1.614 & $0.942-2.768$ & 0.081 & - & - & - \\
\hline Grade & G2/G3 & 4.360 & $2.408-7.894$ & $<0.001$ & 1.555 & $0.790-3.062$ & 0.201 \\
\hline First presenting symptom & & 0.934 & $0.835-1.043$ & 0.225 & - & - & - \\
\hline First presenting symptom longest duration & & 0.832 & $0.701-0.987$ & 0.035 & 0.832 & $0.688-1.006$ & 0.057 \\
\hline Radiation therapy & & 0.790 & $0.516-1.210$ & 0.279 & - & - & - \\
\hline Seizure history & & 1.243 & $0.803-1.923$ & 0.329 & - & - & - \\
\hline Diagnoses & & 0.695 & $0.573-0.843$ & $<0.001$ & 0.703 & $0.572-0.865$ & $<0.001$ \\
\hline Type & Primary/ Recurrent & 1.401 & $0.436-4.497$ & 0.571 & - & - & - \\
\hline IDH1 mutation status & & 0.959 & $0.650-1.417$ & 0.835 & - & - & - \\
\hline RiskScore & & 1.086 & $1.063-1.110$ & $<0.001$ & 1.050 & $1.024-1.077$ & $<0.001$ \\
\hline \multicolumn{8}{|l|}{ Validation cohort $(n=236)$} \\
\hline Age & & 1.047 & $1.025-1.069$ & $<0.001$ & 1.051 & $1.028-1.074$ & $<0.001$ \\
\hline Gender & Female/Male & 0.747 & $0.452-1.234$ & 0.254 & - & - & - \\
\hline Grade & G2/G3 & 2.799 & $1.613-4.857$ & $<0.001$ & 2.279 & $1.216-4.273$ & 0.010 \\
\hline First presenting symptom & & 0.948 & $0.849-1.059$ & 0.348 & - & - & - \\
\hline First presenting symptom longest duration & & 0.884 & $0.749-1.045$ & 0.148 & - & - & - \\
\hline Radiation therapy & & 1.043 & $0.695-1.565$ & 0.839 & - & - & - \\
\hline Seizure history & & 1.325 & $0.851-2.064$ & 0.213 & - & - & - \\
\hline Diagnoses & Primary/ Recurrent & 0.778 & $0.658-0.920$ & 0.003 & 0.859 & $0.707-1.044$ & 0.127 \\
\hline Type & & 1.183 & $0.528-2.651$ & 0.683 & - & - & - \\
\hline IDH1 mutation status & & 1.528 & $0.924-2.528$ & 0.099 & - & - & - \\
\hline RiskScore & & 1.027 & $1.016-1.038$ & $<0.001$ & 1.016 & $1.004-1.029$ & 0.011 \\
\hline \multicolumn{8}{|l|}{ TCGA cohort $(n=476)$} \\
\hline Age & & 1.057 & $1.042-1.073$ & $<0.001$ & 1.061 & $1.044-1.078$ & $<0.001$ \\
\hline Gender & Female/Male & 1.066 & $0.746-1.524$ & 0.726 & - & - & - \\
\hline Grade & G2/G3 & 3.338 & $2.254-4.944$ & $<0.001$ & 1.898 & $1.216-2.960$ & 0.005 \\
\hline First presenting symptom & & 0.944 & $0.873-1.020$ & 0.144 & - & - & - \\
\hline First presenting symptom longest duration & & 0.858 & $0.762-0.966$ & 0.011 & 0.908 & $0.802-1.028$ & 0.129 \\
\hline Radiation therapy & Primary/ Recurrent & 0.923 & $0.688-1.237$ & 0.590 & - & - & - \\
\hline Seizure history & & 1.282 & $0.940-1.749$ & 0.117 & - & - & - \\
\hline Diagnoses & & 0.745 & $0.658-0.844$ & $<0.001$ & 0.799 & $0.694-0.919$ & 0.002 \\
\hline Type & & 1.233 & $0.642-2.368$ & 0.529 & - & - & - \\
\hline IDH1 mutation status & & 1.190 & $0.875-1.618$ & 0.268 & - & - & - \\
\hline RiskScore & & 1.033 & $1.025-1.041$ & $<0.001$ & 1.019 & $1.010-1.029$ & $<0.001$ \\
\hline
\end{tabular}

Next, expression levels of some IncRNAs (ARHGEF26-AS1, PCED1B-AS1, PRKCQ-AS1, LINC00957) in the autophagy-related IncRNA signature were analyzed in a CGGA dataset. ARHGEF26-AS1 was found to be significantly associated with age ( 40 years and >= 40 years), tumor grade, IDH1 mutation and 1p19q codeletion status, chemotherapy status, radiotherapy status and PRS type $(P<0.05)$ (Fig. 7A). PCED1B-AS1 was evidently associated with tumor grade (Grade III vs. IV), IDH1 mutation and 1p19q codeletion status ( $<<0.05)$ (Fig. 7B). PRKCQ-AS1 was significantly associated with tumor grade (Grade III vs. IV), IDH1 mutation and MGMTp codeletion status $(\mathrm{P}<0.05)$ (Fig. 7C). LINC00957 was significantly correlated to IDH1 mutation and chromosome 1p19q codeletion status $(P<0.05)$ (Fig. 7D). Similar findings were also noted in a GEO microarray, GSE16011. As showed in Fig. 7E, a significant association was noticed 
between PRKCQ-AS1 expression and age or IDH1 (R132) mutation status ( $p<0.001)$ (Fig. 7E). In addition, KM curves in CGGA dataset identified a higher OS of patients highly expressing ARHGEF26-AS1 and PRKCQ-AS1 $(P<0.05)$, while a lower OS of patients highly expressing PCED1B-AS1 ( $<<0.05)$, compared to

corresponding control groups. In GSE16011, patients highly expressing PRKCQ-AS1 possessed a considerably higher OS relative to patients lowly expressing PRKCQ-AS1 ( $<<0.001)$ (Supplementary Fig. 3A-E).

\subsection{GSEA}

To probe into the underlying biological behavior of autophagy-related IncRNAs in LGG, an analysis for autophagy biological processes, negative regulation of autophagy, GO and KEGG analysis in GSEA was implemented, and the most significantly enriched biological functions (INES|>1, NOM P-value $<0.05$ and FDR q-value $<0.25$ ) were selected. Results showed that pathways related to cancer and autophagy were highly different in high- and low-risk cohorts. Besides, the high-risk group was profoundly related to reactome autophagy, negative regulation of autophagy, negative regulation of mitotic cell cycle, apoptotic process involved in morphogenesis, negative regulation of cell cycle process, DNA damage response signal transduction by p53 class mediator, integrin-mediated signaling pathway, apoptosis, antigen processing and presentation, pathways in cancer, JAK/STAT signaling pathway, p53 signaling pathway, small cell lung cancer and pancreatic cancer, while the low-risk group was closely related to ionotropic glutamate receptor signaling pathway and GABA receptor binding (Fig. 8A, Table 4). It was suggestive that these autophagy-related IncRNAs were involved in the pathways related to carcinogenic activation and autophagy in LGG, providing clues for targeted therapy of LGG. We also revealed 492 differential target mRNAs regulated by the identified IncRNAs between low and high risk groups.( Supplementary Table 1) 


\begin{tabular}{|c|c|c|c|c|c|c|c|}
\hline MSigDB collection & Gene set name & Size & ES & NES & $\begin{array}{l}\text { NOM } \\
\text { P-val }\end{array}$ & $\begin{array}{l}\text { FDRq- } \\
\text { val }\end{array}$ & $\begin{array}{l}F \\
V_{i}\end{array}$ \\
\hline & KEGG_PATHWAYS_IN_CANCER & 324 & 0.449 & 1.867 & 0 & 0.016 & 0 \\
\hline & KEGG_JAK_STAT_SIGNALING_PATHWAY & 155 & 0.533 & 1.918 & 0.002 & 0.009 & 0 \\
\hline & KEGG_P53_SIGNALING_PATHWAY & 68 & 0.588 & 1.958 & 0 & 0.008 & 0 \\
\hline & KEGG_SMALL_CELL_LUNG_CANCER & 84 & 0.557 & 1.969 & 0 & 0.007 & 0 \\
\hline & KEGG_PANCREATIC_CANCER & 69 & 0.503 & 1.853 & 0.004 & 0.017 & 0 \\
\hline
\end{tabular}

ES: enrichment score; NES: normalized enrichment score; NOM: nominal; FDR: false discovery rate; FWER: familywise-error rate;Gene sets with NOM P-value < 0.25 were considered as significantly enriched.

\section{Discussion}

Autophagy involves in the evolution process of eukaryotic cells which are highly conservative, and functions in the degradation almost in all cells from yeast to mammals[35]. Autophagy disorders are associated with many diseases. LncRNA as a novel autophagy-regulating molecule acts on gene expression at transcriptional and post-transcriptional levels[36]. It acts as a scaffold for recruitment of chromatin modifiers in transcriptional regulation, which induces epigenetic silencing and participates in regulating histone modification, DNA methylation and chromosome remodeling, enhancing gene transcription, and regulating gene transcription by altering the subcellular localization of transcription factors[37]. For example, IncRNA 2810403D21Rik/Mirf as a regulator of autophagy functions via the miR-26a-USP15 axis, to inhibit autophagy and induce myocardial injury by reducing intrinsic cardioprotective activity. While silencing IncRNA 2810403D21Rik/Mirf can reduce the H2O2-induced myocardial injury, which can be reversed under miR-26a or 3-MA knockout by AMO-26a to inhibit autophagy in NMCM[38]. In the post-transcriptional level, IncRNA as a precursor of small RNA assists in mRNA stabilizing or degrading in a form of small RNA regulating mRNA processing and stability[39]. As previous studies shown, IncRNA HOTAIRM1 functions on autophagy and tumor protein PMLRARA degradation during bone marrow cell differentiation arrest, and down-regulated HOTAIRM1 can inhibit the PML-RARA degradation induced by all-trans retinoic acid in acute promyelocytic leukemia (APL) cells and then block the differentiation from early promyelocytes to granulocytes[40].

Most studies on IncRNA focus on specific genes involved in autophagy, while autophagy-related IncRNA prognostic in LGG patients is a less studied field. We here established an autophagy-related IncRNA signature in LGG which was prognostic. In our research, co-expression of ATGs and IncRNAs was firstly analyzed to screen autophagy-related IncRNAs, followed by univariate, Lasso, and multivariate Cox analyses to identify 9 prognostic autophagy-related IncRNAs, including AL136964.1, ARHGEF26-AS1, PCED1B-AS1, AC104072.1, PRKCQ-AS1, LINC00957, AC125616.1, PSMB8-AS1, and AC087741.1. These 9 IncRNAs may be prognostic markers and therapeutic targets for LGG patients. Among the 9 genes, 5 of them (ARHGEF26-AS1, PCED1B-AS1, PRKCQ-AS1, LINC00957, and PSMB8-AS1) have been proven of cancer relevant. (1) ARHGEF26-AS1 (ARHGEF26 antisense RNA 1) as the antisense RNA of ARHGEF26 is reported to show a significant correlation with OS of colon cancer $(P<0.05)$ [41]. In addition, ARHGEF26-AS1 acts as a central gene in the ceRNA network to indicate the prognosis of intrahepatic cholangiocarcinoma and colon cancer[42]. (2) PCED1B-AS1 presents profoundly up-regulated expression in clear cell renal cell carcinoma (ccRCC) tissues and cell lines, which indicates poor prognosis of patients. Besides, it can mediate ccRCC cell proliferative and migratory abilities as well as epithelial-mesenchymal transition (EMT) process, and promote the expression of ZEB1 by inhibiting miR-484[43]. Recent studies have shown that PCED1B-AS1 induces immunosuppression and increases the expression and function of PD-Ls in liver cancer as a sponge for miR-194-5p. It also promotes the proliferation of transplanted tumor cell in nude mice, along with increased colony formation and tumor formation in vivo, and inhibited cell apoptosis[44]. PCED1B-AS1 can bind HIF-1a mRNA 5'-UTR in a direct way and to enhance HIF-1a translation, resulting in the increase of HIF-1a proteins, thus advancing the Warburg effect and tumorigenesis of glioblastoma[45]. In addition, PCED1B-AS1 can activate glioma cells to proliferate while inhibiting apoptosis with miR-194-5p/PCED1B axis[46]. Other studies found that PCED1B-AS1 modulates macrophage apoptosis and autophagy in active tuberculosis via targeting miR-155[47]. (3) LncRNA PRKCQ-AS1 is proven prognostic in colorectal cancer (CRC) patients as a risk factor[48]. It is concentrated in the cytoplasm of CRC cells and reversely regulates miR-1287-5p expression, which indicates adverse outcomes[49]. Recent studies have also shown that PRKCQ- 
AS1, as an immune-related IncRNA, provides a reliable prognostic indicator for glioblastoma patients[50]. (4) LINC00957 is significantly related to poor OS of CRC patients upon increased expression ( $\mathrm{P}<0.05$ ), and inhibiting LINC00957 expression can reverse 5-FU resistance via P-gP down regulation[51]. (5) PSMB8AS1 is involved in pancreatic cancer progression with miR-382-3p/STAT1/PD-L1 axis, while knockout of PSMB8-AS1 leads to weakened cell proliferation, migration, invasion and EMT, while increased cell apoptosis[52]. In addition, PSMB8-AS1 as a ceRNA for miR-22-3p regulates DDIT4 in glioblastoma. Contrarily, over expression of DDIT4 could antagonize the effects of PSMB8-AS1 on proliferation, apoptosis and radioresistance of glioblastoma cells[53]. Other studies showed that the activation of PSMB8-AS1 by transcription factor ELK1 advances cell proliferation of glioma by regulating miR-574-5p/RAB10, while silencing PSMB8-AS1 inhibits the proliferation[54]. For the other 4 autophagy-related IncRNAs (AL136964.1, AC104072.1, AC125616.1 and AC087741.1), there is no report on their prognostic role in cancer. It is necessary to conduct more research to identify the mechanism behind the effect of these IncRNAs on the prognosis of LGG individuals via autophagy.

The IncRNA signature we identified could be used to effectively predict the survival of patients suffering from LGG. According to the expression of the 9 signature IncRNAs, each LGG patient was conferred a Riskscore, based on which two risk cohorts were generated (cutoff: median Riskscore). As analyzed, LGG samples with high Riskscores had poorer survival compared to those with low Riskscores $(P<0.05)$. ROC analysis verified the prognostic accuracy of the IncRNA signature in LGG patients. Besides, the signature-based Riskscore was identified independently prognostic in multivariate analysis ( $P<0.05)$, and presented more reliable prognostic performance relative to other traditional clinical indicators. Nomogram, C-index, calibration curve and DCA curve are effective and reliable clinical tools for survival prediction of cancer patients. Here, we established a robust nomogram involving multiple clinical variables (age, gender, diagnoses, grade, radiation_therapy, seizure_history, type, IDH1_mutation_status) and the signature-based Riskscore which helps improve the prognosis prediction of LGG patients. Patients of a higher age ( > = 40 years) or higher tumor grade generally show poor cancer prognosis, which is consistent with our results. Moreover, the calibration curve displayed a similar result between the actual and predicted 3-year and 5-year survival rates based on the nomogram. GSEA results suggested that the 9 autophagy-related IncRNAs may negatively regulate autophagy and cancer-related signaling pathways, and play a vital part in the pathogenesis of LGG.

However, there remain some limitations that need to be concerned. Firstly, data analyzed here were from TCGA, GEO, and CGGA databases, which requires a further validation in other prospective cohorts to ensure the robustness of the signature we identified. Secondly, the potential molecular correlation between autophagy-related IncRNAs and autophagy need further study. Finally, the role and mechanism behind these autophagy-related IncRNAs in LGG call for a further investigation.

In conculsion, the prognostic signature involving autophagy-related IncRNAs we identified here was accurate in survival prediction of LGG sufferers, which show great clinical application potential in individualized prognosis and treatment. Remarkable associations were revealed of the 9 signature IncRNAs with survival of LGG cases, and a high performance of the signature-based Riskscore in distinguishing patients at different risks was uncovered, suggesting the independent role of the signature. The autophagy-related IncRNAs and their signature, therefore, are potential molecular biomarkers and therapeutic targets of LGG.

\section{Declarations}

\section{Funding}

This study was not funded by anybody.

\section{Author Contributions}

Aierpati Maimaiti \& Mirezhati Tuerhong: Conceptualization, Methodology, Validation, Investigation, Supervision, Software, Visualization, Writing - original draft, Writing- Reviewing and Editing. Yongxin Wang: Validation, Supervision, Investigation. Maimaitili Aisha: Visualization, Methodology. Lei Jiang: Data curation, Software, Validation. Xixian Wang: Methodology, Formal analysis, Visualization. Yusufu Mahemuti: Software, Validation. Yirizhati Aili: Data curation, Visualization, Validation. Zhaohai Feng: Investigation,Validation. Maimaitijiang Kasimu: Investigation, Writing- Reviewing and Editing.

\section{Data Availability}

All the data generated or analyzed during this study have been included in this article.

\section{Declarations}

\section{Conflict of Interest}

The authors declare no conflicts of interest.

\section{Ethics approval and consent to participate}

Not required to this study.

\section{Patient consent for publication}

Not required.

\section{References}


1. Li Y, Deng G, Qi Y, Zhang H, Jiang H, Geng R, Ye Z, Liu B, Chen Q (2020) Downregulation of LUZP2 Is Correlated with Poor Prognosis of Low-Grade Glioma. BioMed research international 2020:9716720. doi:10.1155/2020/9716720

2. Bao Z, Wang Y, Wang Q, Fang S, Shan X, Wang J, Jiang T (2021) Intratumor heterogeneity, microenvironment, and mechanisms of drug resistance in glioma recurrence and evolution. Frontiers of medicine. doi:10.1007/s11684-020-0760-2

3. Bandopadhayay P, Bergthold G, London W, Goumnerova L, Morales La Madrid A, Marcus K, Guo D, Ullrich N, Robison N, Chi S, Beroukhim R, Kieran M, Manley P (2014) Long-term outcome of 4,040 children diagnosed with pediatric low-grade gliomas: an analysis of the Surveillance Epidemiology and End Results (SEER) database. Pediatric blood cancer 61:1173-1179. doi:10.1002/pbc.24958

4. Lin H, Patel S, Affleck V, Wilson I, Turnbull D, Joshi A, Maxwell R, Stoll E (2017) Fatty acid oxidation is required for the respiration and proliferation of malignant glioma cells. Neurooncology 19:43-54. doi:10.1093/neuonc/now128

5. Duffau H, Taillandier L (2015) New concepts in the management of diffuse low-grade glioma: Proposal of a multistage and individualized therapeutic approach. Neurooncology 17:332-342. doi:10.1093/neuonc/nou153

6. Xue W, Zhang J, Tong H, Xie T, Chen X, Zhou B, Wu P, Zhong P, Du X, Guo Y, Yang Y, Liu H, Fang J, Wang S, Wu H, Xu K, Zhang W (2020) Effects of BMPER, CXCL10, and HOXA9 on Neovascularization During Early-Growth Stage of Primary High-Grade Glioma and Their Corresponding MRI Biomarkers. Frontiers in oncology 10:711. doi:10.3389/fonc.2020.00711

7. Jiang L, Xiao C, Xu Q, Sun J, Chen H, Chen Y, Yin X (2017) Analysis of DTI-Derived Tensor Metrics in Differential Diagnosis between Low-grade and Highgrade Gliomas. Front Aging Neurosci 9:271. doi:10.3389/fnagi.2017.00271

8. Yang Y, Liu X, Cheng L, Li L, Wei Z, Wang Z, Han G, Wan X, Wang Z, Zhang J, Chen C (2020) Tumor Suppressor microRNA-138 Suppresses Low-Grade Glioma Development and Metastasis via Regulating IGF2BP2. OncoTargets therapy 13:2247-2260. doi:10.2147/ott.S232795

9. Zhao J, Wang L, Wei B (2020) Identification and Validation of an Energy Metabolism-Related IncRNA-mRNA Signature for Lower-Grade Glioma. Biomed Res Int 2020:3708231. doi:10.1155/2020/3708231

10. Louis D, Perry A, Reifenberger G, von Deimling A, Figarella-Branger D, Cavenee W, Ohgaki H, Wiestler O, Kleihues P, Ellison D (2016) The 2016 World Health Organization Classification of Tumors of the Central Nervous System: a summary. Acta Neuropathol 131:803-820. doi:10.1007/s00401-016-1545-1

11. Zhang M, Wang X, Chen X, Zhang Q, Hong J (2020) Novel Immune-Related Gene Signature for Risk Stratification and Prognosis of Survival in LowerGrade Glioma. Frontiers in genetics 11:363. doi:10.3389/fgene.2020.00363

12. Fisher M, Jones D, Li Y, Guo X, Sonawane P, Waanders A, Phillips J, Weiss W, Resnick A, Gosline S, Banerjee J, Guinney J, Gnekow A, Kandels D, Foreman N, Korshunov A, Ryzhova M, Massimi L, Gururangan S, Kieran M, Wang Z, Fouladi M, Sato M, Øra I, Holm S, Markham S, Beck P, Jäger N, Wittmann A, Sommerkamp A, Sahm F, Pfister S, Gutmann D (2021) Integrated molecular and clinical analysis of low-grade gliomas in children with neurofibromatosis type 1 (NF1). Acta Neuropathol 141:605-617. doi:10.1007/s00401-021-02276-5

13. Tu Z, Wu L, Wang P, Hu Q, Tao C, Li K, Huang K, Zhu X (2020) N6-Methylandenosine-Related IncRNAs Are Potential Biomarkers for Predicting the Overall Survival of Lower-Grade Glioma Patients. Frontiers in cell developmental biology 8:642. doi:10.3389/fcell.2020.00642

14. Liu W, Li C, Xu W, Liu X, Tang H, Huang H (2020) Genome-wide analyses of the prognosis-related mRNA alternative splicing landscape and novel splicing factors based on large-scale low grade glioma cohort. Aging 12:13684-13700. doi:10.18632/aging.103491

15. Xie C, Liu X, Sham K, Lai J, Cheng C (2014) Silencing of EEF2K (eukaryotic elongation factor-2 kinase) reveals AMPK-ULK1-dependent autophagy in colon cancer cells. Autophagy 10:1495-1508. doi:10.4161/auto.29164

16. Jutten B, Keulers T, Peeters H, Schaaf M, Savelkouls K, Compter I, Clarijs R, Schijns O, Ackermans L, Teernstra O, Zonneveld M, Colaris R, Dubois L, Vooijs M, Bussink J, Sotelo J, Theys J, Lammering G, Rouschop K (2018) EGFRvIll expression triggers a metabolic dependency and therapeutic vulnerability sensitive to autophagy inhibition. Autophagy 14:283-295. doi:10.1080/15548627.2017.1409926

17. Wang H, Liu Y, Wang D, Xu Y, Dong R, Yang Y, Lv Q, Chen X, Zhang Z (2019) The Upstream Pathway of mTOR-Mediated Autophagy in Liver Diseases. Cells 8. doi:10.3390/cells8121597

18. Gu Y, Han J, Jiang C, Zhang Y (2020) Biomarkers, oxidative stress and autophagy in skin aging. Ageing Res Rev 59:101036. doi:10.1016/j.arr.2020.101036

19. Su S, Wang X, Xi X, Zhu L, Chen Q, Zhang H, Qin Y, Yang B, Che N, Cao H, Zhong W, Wang B (2021) Phellodendrine promotes autophagy by regulating the AMPK/mTOR pathway and treats ulcerative colitis. J Cell Mol Med. doi:10.1111/jcmm.16587

20. Zhang Y, Gao R, Zhang L, Geng Y, Chen Q, Chen X, Liu X, Mu X, Ding Y, Wang Y, He J (2021) AMPK/mTOR downregulated autophagy enhances aberrant endometrial decidualization in folate-deficient pregnant mice. Journal of cellular physiology. doi:10.1002/jcp.30408

21. Mukhopadhyay S, Panda P, Sinha N, Das D, Bhutia S (2014) Autophagy and apoptosis: where do they meet? Apoptosis: an international journal on programmed cell death 19:555 - 66. doi: 10.1007/s10495-014-0967-2

22. Mariño G, Niso-Santano M, Baehrecke E, Kroemer G (2014) Self-consumption: the interplay of autophagy and apoptosis. Nat Rev Mol Cell Biol 15:81-94. doi:10.1038/nrm3735

23. Greabu M, Giampieri F, Imre M, Mohora M, Totan A, Pituru S, Ionescu E (2020) Autophagy, One of the Main Steps in Periodontitis Pathogenesis and Evolution. Molecules (Basel, Switzerland) 25. doi: 10.3390/molecules25184338

24. Zois C, Koukourakis M (2009) Radiation-induced autophagy in normal and cancer cells: towards novel cytoprotection and radio-sensitization policies? Autophagy 5:442 - 50. doi: 10.4161/auto.5.4.7667

25. Salminen A, Kaarniranta K, Kauppinen A (2013) Beclin 1 interactome controls the crosstalk between apoptosis, autophagy and inflammasome activation: impact on the aging process. Ageing Res Rev 12:520-534. doi:10.1016/j.arr.2012.11.004

Page $13 / 21$ 
26. Vlahakis A, Debnath J (2017) The Interconnections between Autophagy and Integrin-Mediated Cell Adhesion. Journal of molecular biology 429:515-530. doi:10.1016/j.jmb.2016.11.027

27. Shen H, Wang L, Xiong J, Ren C, Gao C, Ding W, Zhu D, Ma D, Wang H (2019) Long non-coding RNA CCAT1 promotes cervical cancer cell proliferation and invasion by regulating the miR-181a-5p/MMP14 axis. Cell Cycle 18:1110-1121. doi:10.1080/15384101.2019.1609829

28. Liu L, Zhu S, Jiang X, Ren J, Lin Y, Zhang N, Tong M, Zhang H, Zheng W, Fu H, Luo H, Lin L, Yan J, Yang T (2017) LncRNA Expression in CD4 + T Cells in Neurosyphilis Patients. Front Cell Infect Microbiol 7:461. doi:10.3389/fcimb.2017.00461

29. Zhao W, Lin X, Han H, Zhang H, Li X, Jiang C, Feng M (2021) Long noncoding RNA H19 contributes to the proliferation and autophagy of glioma cells through mTOR/ULK1 pathway. Neuroreport 32:352-358. doi:10.1097/wnr.0000000000001602

30. Lv Q, Wang L, Li D, Lin Q, Shen X, Liu H, Li M, Ji Y, Qin C, Chen S (2020) Knockdown IncRNA DLEU1 Inhibits Gliomas Progression and Promotes Temozolomide Chemosensitivity by Regulating Autophagy. Front Pharmacol 11:560543. doi:10.3389/fphar.2020.560543

31. Zheng J, Wang B, Zheng R, Zhang J, Huang C, Zheng R, Huang Z, Qiu W, Liu M, Yang K, Mao Z, Ji A, Yuan Y (2020) Linc-RA1 inhibits autophagy and promotes radioresistance by preventing H2Bub1/USP44 combination in glioma cells. Cell death disease 11:758. doi:10.1038/s41419-020-02977-X

32. Ma R, Zhang B, Zhang Z, Deng Q (2020) LncRNA MALAT1 knockdown inhibits cell migration and invasion by suppressing autophagy through miR384/GOLM1 axis in glioma. Eur Rev Med Pharmacol Sci 24:2601-2615. doi:10.26355/eurrev_202003_20529

33. Liu C, Fu H, Liu X, Lei Q, Zhang Y, She X, Liu Q, Liu Q, Sun Y, Li G, Wu M (2018) LINC00470 Coordinates the Epigenetic Regulation of ELFN2 to Distract GBM Cell Autophagy. Molecular therapy: the journal of the American Society of Gene Therapy 26:2267-2281. doi:10.1016/j.ymthe.2018.06.019

34. Charoentong P, Finotello F, Angelova M, Mayer C, Efremova M, Rieder D, Hackl H, Trajanoski Z (2017) Pan-cancer Immunogenomic Analyses Reveal Genotype-Immunophenotype Relationships and Predictors of Response to Checkpoint Blockade. Cell reports 18:248-262. doi:10.1016/j.celrep.2016.12.019

35. Wei J, Ge X, Tang Y, Qian Y, Lu W, Jiang K, Fang Y, Hwang M, Fu D, Xiao Q, Ding K (2020) An Autophagy-Related Long Noncoding RNA Signature Contributes to Poor Prognosis in Colorectal Cancer. Journal of oncology 2020:4728947. doi:10.1155/2020/4728947

36. Guo J, Li Y, Duan H, Yuan L (2019) LncRNA TUBA4B functions as a competitive endogenous RNA to inhibit gastric cancer progression by elevating PTEN via sponging miR-214 and miR-216a/b. Cancer cell international 19:156. doi:10.1186/s12935-019-0879-x

37. Pellecchia S, Sepe R, Decaussin-Petrucci M, Ivan C, Shimizu M, Coppola C, Testa D, Calin G, Fusco A, Pallante P (2020) Prader Willi/Angelman Region RNA5The Long Non-Coding RNA Is Downregulated in Anaplastic Thyroid Carcinomas Where It Acts as a Tumor Suppressor by Reducing EZH2 Activity. Cancers 12. doi: 10.3390/cancers12010235

38. Liang H, Su X, Wu Q, Shan H, Lv L, Yu T, Zhao X, Sun J, Yang R, Zhang L, Yan H, Zhou Y, Li X, Du Z, Shan H (2020) 2810403D21Rik/MirfLncRNA promotes ischemic myocardial injury by regulating autophagy through targeting. Autophagy 16:1077-1091. doi:10.1080/15548627.2019.1659610

39. Zheng J, Pang C, Du W, Wang L, Sun L, Xing Z (2020) An allele of rs619586 polymorphism in MALAT1 alters the invasiveness of meningioma via modulating the expression of collagen type $V$ alpha (COL5A1). J Cell Mol Med 24:10223-10232. doi:10.1111/jcmm.15637

40. Chen Z, Wang W, Huang W, Fang K, Sun Y, Liu S, Luo X, Chen Y (2017) The IncRNA HOTAIRM1 regulates the degradation of PML-RARA oncoprotein and myeloid cell differentiation by enhancing the autophagy pathway. Cell death differentiation 24:212-224. doi:10.1038/cdd.2016.111

41. Gao Z, Fu P, Yu Z, Zhen F, Gu Y (2019) Comprehensive Analysis of IncRNA-miRNA- mRNA Network Ascertains Prognostic Factors in Patients with Colon Cancer. Technology in cancer research treatment 18:1533033819853237. doi:10.1177/1533033819853237

42. Zhou D, Gao B, Yang Q, Kong Y, Wang W (2019) Integrative Analysis of ceRNA Network Reveals Functional IncRNAs in Intrahepatic Cholangiocarcinoma. BioMed research international 2019:2601271. doi:10.1155/2019/2601271

43. Qin J, Zhu T, Wu W, Chen H, He Y (2021) Long Non-Coding RNA PCED1B-AS1 Promotes the Progression of Clear Cell Renal Cell Carcinoma Through miR484/ZEB1 Axis. OncoTargets therapy 14:393-402. doi:10.2147/ott.S270149

44. Fan F, Chen K, Lu X, Li A, Liu C, Wu B (2020) Dual targeting of PD-L1 and PD-L2 by PCED1B-AS1 via sponging hsa-miR-194-5p induces immunosuppression in hepatocellular carcinoma. Hepatology international. doi:10.1007/s12072-020-10101-6

45. Yao Z, Zhang Q, Guo F, Guo S, Yang B, Liu B, Li P, Li J, Guan S, Liu X (2020) Long Noncoding RNA PCED1B-AS1 Promotes the Warburg Effect and Tumorigenesis by Upregulating HIF-1a in Glioblastoma. Cell Transplant 29:963689720906777. doi:10.1177/0963689720906777

46. Yang J, Yu D, Liu X, Changyong E, Yu S (2020) LncRNA PCED1B-AS1 activates the proliferation and restricts the apoptosis of glioma through cooperating with miR-194-5p/PCED1B axis. Journal of cellular biochemistry 121:1823-1833. doi:10.1002/jcb.29417

47. Li M, Cui J, Niu W, Huang J, Feng T, Sun B, Yao H (2019) Long non-coding PCED1B-AS1 regulates macrophage apoptosis and autophagy by sponging miR-155 in active tuberculosis. Biochem Biophys Res Commun 509:803-809. doi:10.1016/j.bbrc.2019.01.005

48. Shademan M, Naseri Salanghuch A, Zare K, Zahedi M, Foroughi M, Akhavan Rezayat K, Mosannen Mozaffari H, Ghaffarzadegan K, Goshayeshi L, Dehghani H (2019) Expression profile analysis of two antisense IncRNAs to improve prognosis prediction of colorectal adenocarcinoma. Cancer cell international 19:278. doi:10.1186/s12935-019-1000-1

49. Cui G, Zhao H, Li L (2020) Long noncoding RNA PRKCQ-AS1 promotes CRC cell proliferation and migration via modulating miR-1287-5p/YBX1 axis. Journal of cellular biochemistry 121:4166-4175. doi:10.1002/jcb.29712

50. Li X, Sun L, Wang X, Wang N, Xu K, Jiang X, Xu S (2021) A Five Immune-Related IncRNA Signature as a Prognostic Target for Glioblastoma. Frontiers in molecular biosciences 8:632837. doi:10.3389/fmolb.2021.632837

51. Zhang L, Li L, Zhang P, Cai Y, Hua D (2019) LINC00957 Acted as Prognostic Marker Was Associated With Fluorouracil Resistance in Human Colorectal Cancer. Frontiers in oncology 9:776. doi:10.3389/fonc.2019.00776

Page $14 / 21$ 
52. Zhang H, Zhu C, He Z, Chen S, Li L, Sun C (2020) LncRNA PSMB8-AS1 contributes to pancreatic cancer progression via modulating miR-3823p/STAT1/PD-L1 axis. Journal of experimental clinical cancer research: CR 39:179. doi:10.1186/s13046-020-01687-8

53. Hu T, Wang F, Han G (2020) LncRNA PSMB8-AS1 acts as ceRNA of miR-22-3p to regulate DDIT4 expression in glioblastoma. Neurosci Lett $728: 134896$. doi:10.1016/j.neulet.2020.134896

54. Shen G, Mao Y, Su Z, Du J, Yu Y, Xu F (2020) PSMB8-AS1 activated by ELK1 promotes cell proliferation in glioma via regulating miR-574-5p/RAB10. Biomedicine pharmacotherapy = Biomedecine pharmacotherapie 122:109658. doi:10.1016/j.biopha.2019.109658

\section{Figures}

A

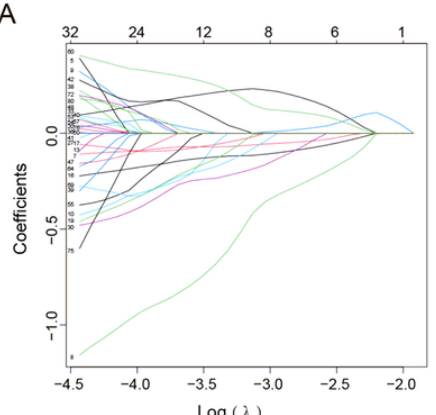

C

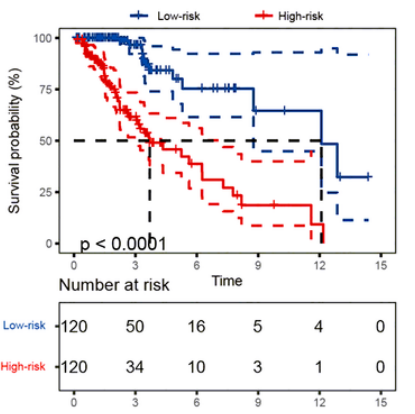

E

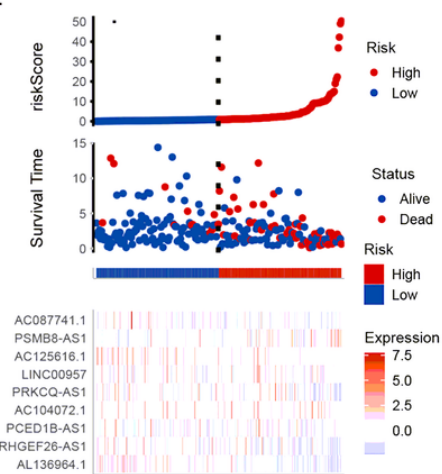

B

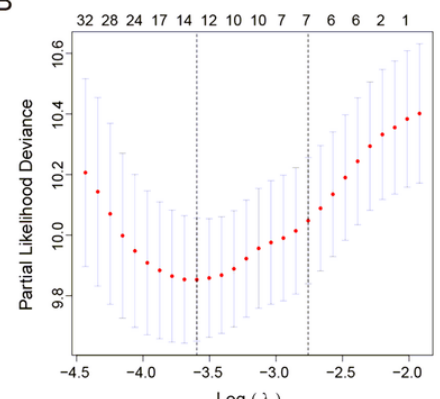

D

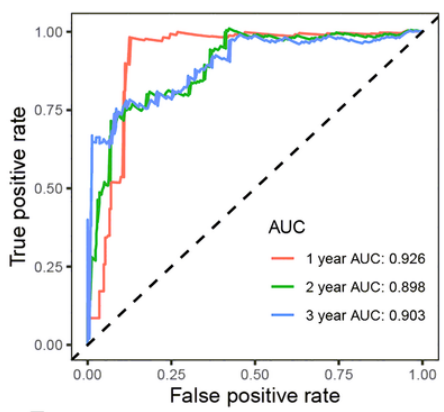

F

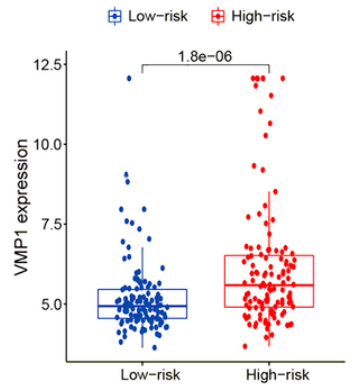

Figure 1

Identification of prognostic autophagy-related IncRNA signature in the training set. A. Lasso-Cox analysis showed 13 autophagy-related IncRNAs highly prognostic. B. The optimal penalty parameter determined by 1000 repeated cross-validation. C. Kaplan-Meier survival estimates of OS in low-risk and high-risk patients in the training set based on the autophagy-related IncRNA signature. D. ROC curves for 1, 2, 3 years of OS in the training set. E. Riskscore distribution, patient survival status and time, and heat map of the 9 autophagy-related IncRNAs in low-risk and high-risk patients in the training set. F. Box plot of VMP1 gene expression in low-risk and high-risk groups of LGG patients. 
A

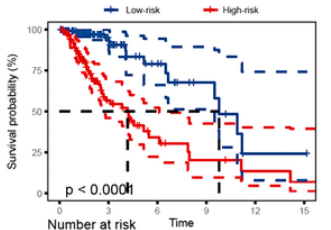

$$
\text { Number at risk }
$$

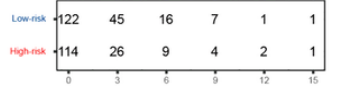

B

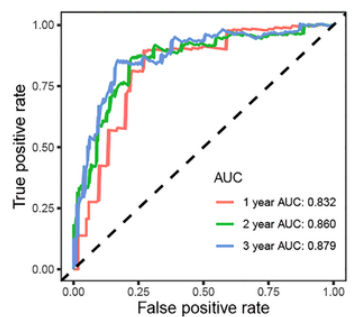

C

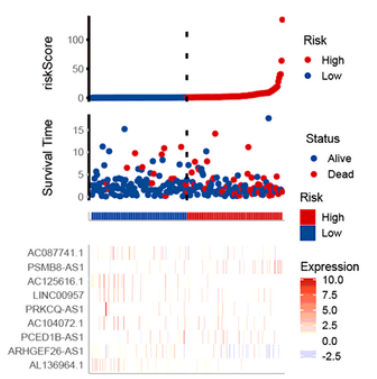

D

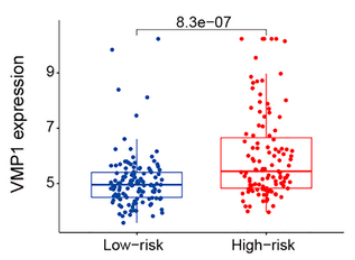

E
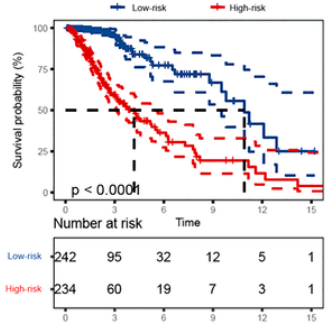

$F$
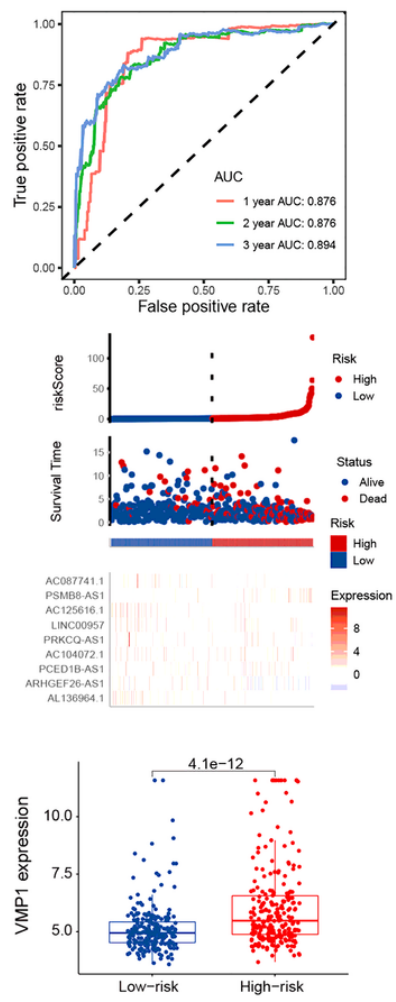

Figure 2

Validation of autophagy-related IncRNA signature in the validation and TCGA sets. A. Kaplan-Meier survival estimates of autophagy-related IncRNAs in predicting OS in low-risk or high-risk patients in the validation set. B. ROC curves for 1, 2 and 3 years in the validation set. C. Riskscore distribution, survival status and time of patients, and heat map of the 9 autophagy-related IncRNAs in low-risk and high-risk patients in the validation group. D. Box plot of VMP1 expression distribution in high-risk and low-risk populations in the validation group. E-H. TCGA validation. 
A
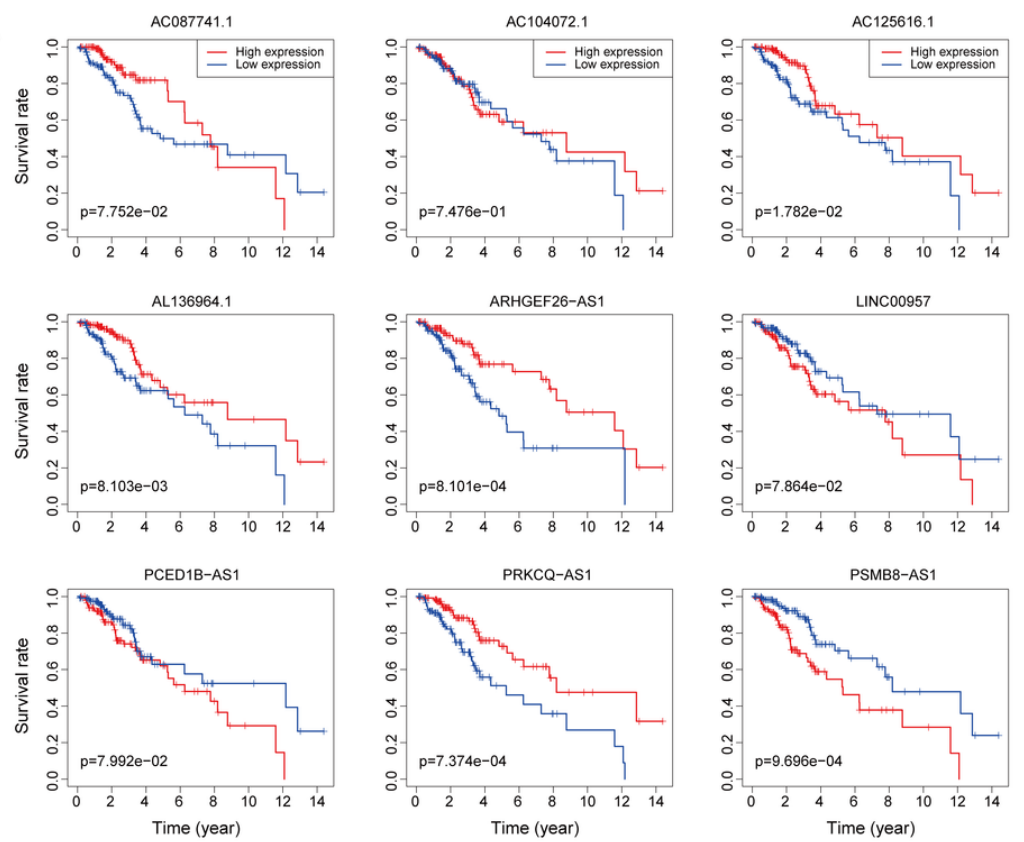

B
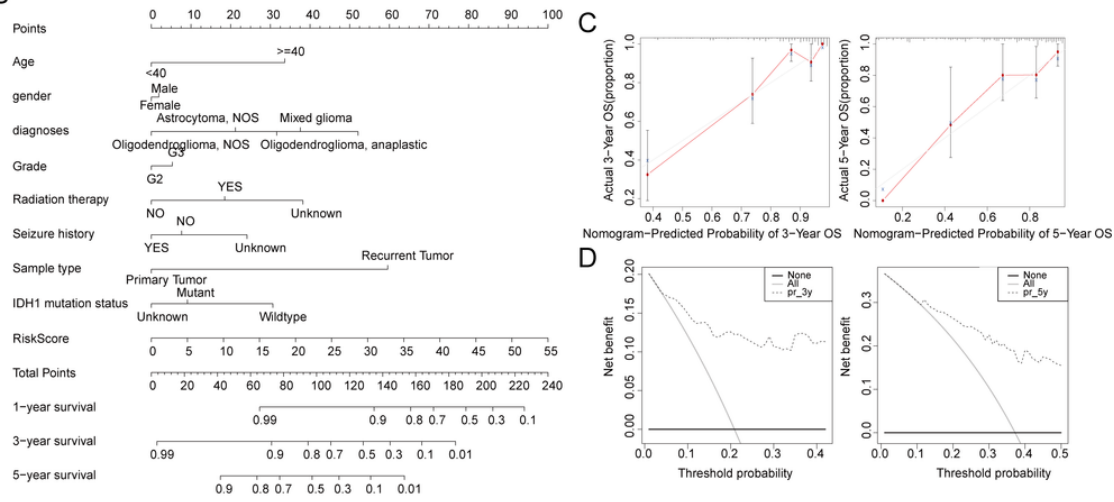

Figure 3

A. Kaplan-Meier survival analysis was performed for OS of LGG patients in the training set based on 9 autophagy-related IncRNAs. B-D. The nomogram constructed based on clinicopathological factors and the IncRNA signature-based Riskscore for prediction of 1-year, 3-year, and 5-year survival of patients in the training set. The nomogram was evaluated using the calibration curve and the DCA curve. 
A
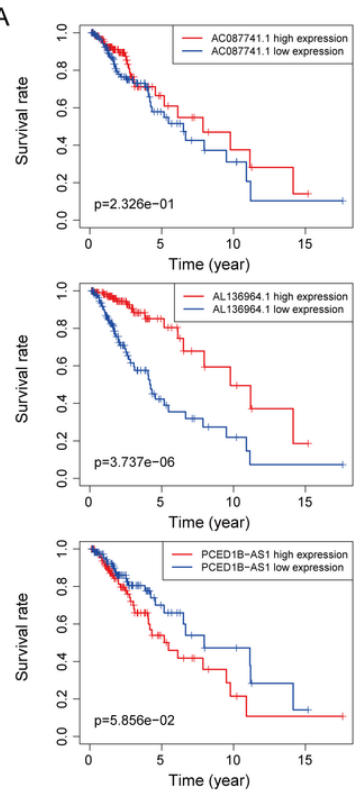

B
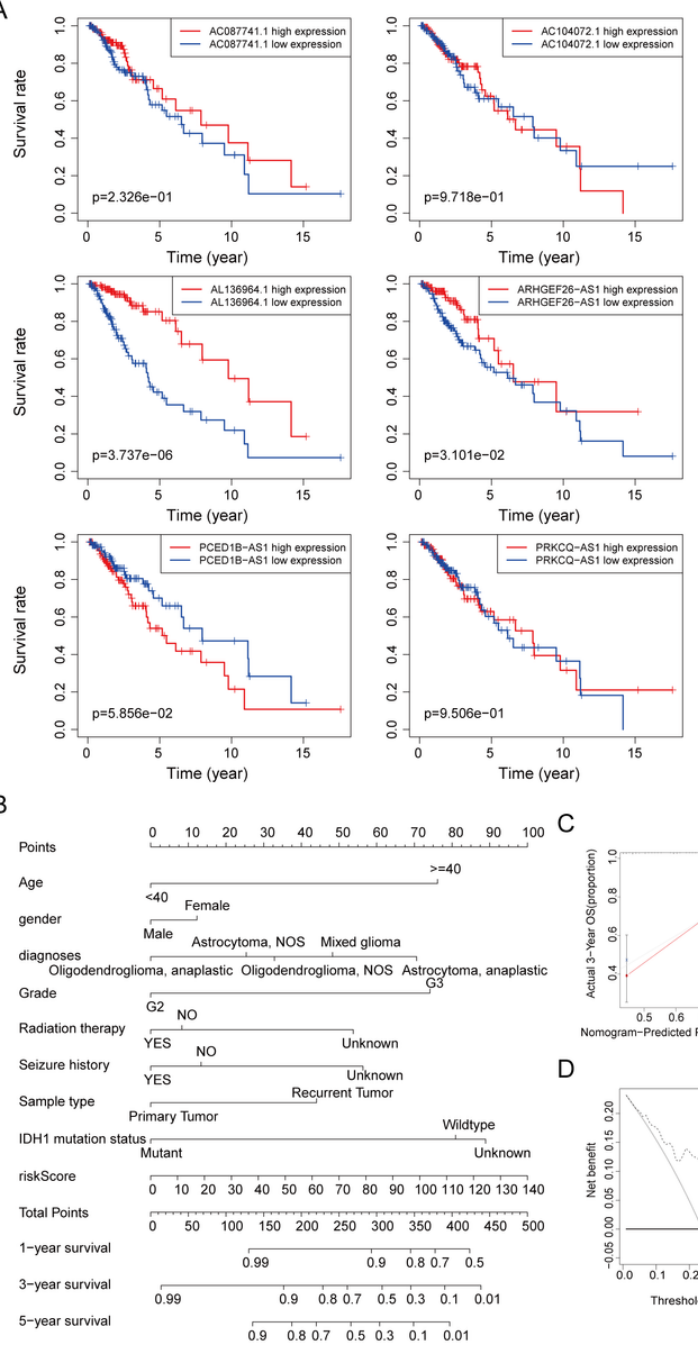

C

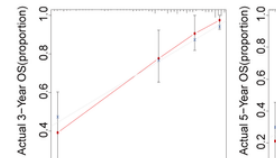

$\begin{array}{lllll}0.5 & 0.6 & 0.7 & 0.8 & 0.9\end{array}$

D

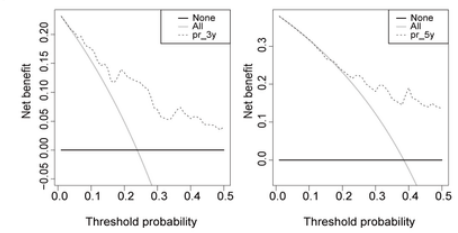

Figure 4

A. Kaplan-Meier survival analysis was performed for OS of LGG patients in the validation set based on 9 autophagy-related IncRNAs. B-D. The nomogram constructed based on clinicopathological factors and the IncRNA signature-based Riskscore for prediction of 1-year, 3-year, and 5-year survival of patients in the validation set. The nomogram was evaluated using the calibration curve and the DCA curve. 
A
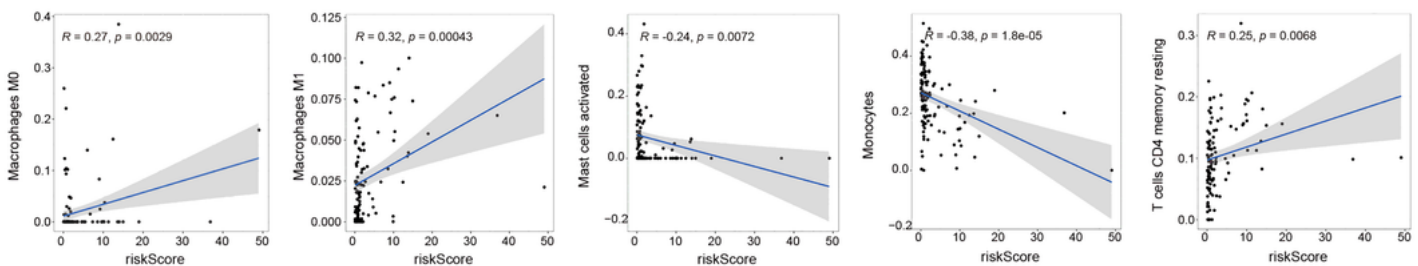

B
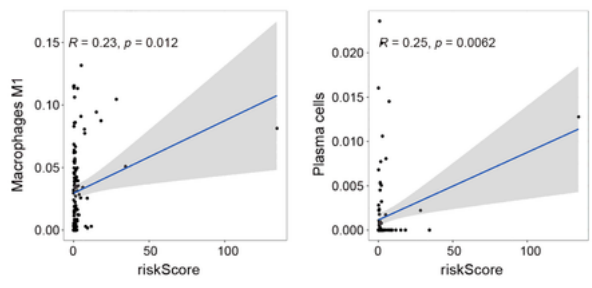

C
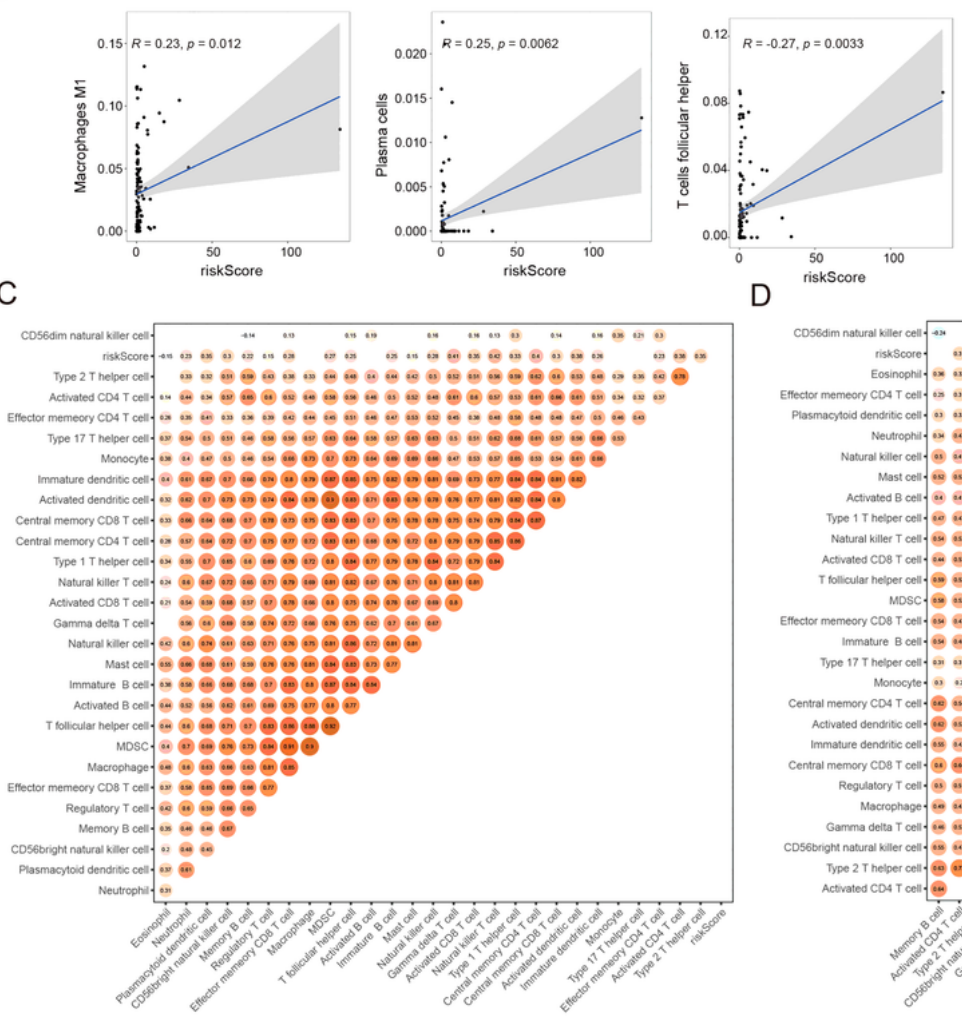

D

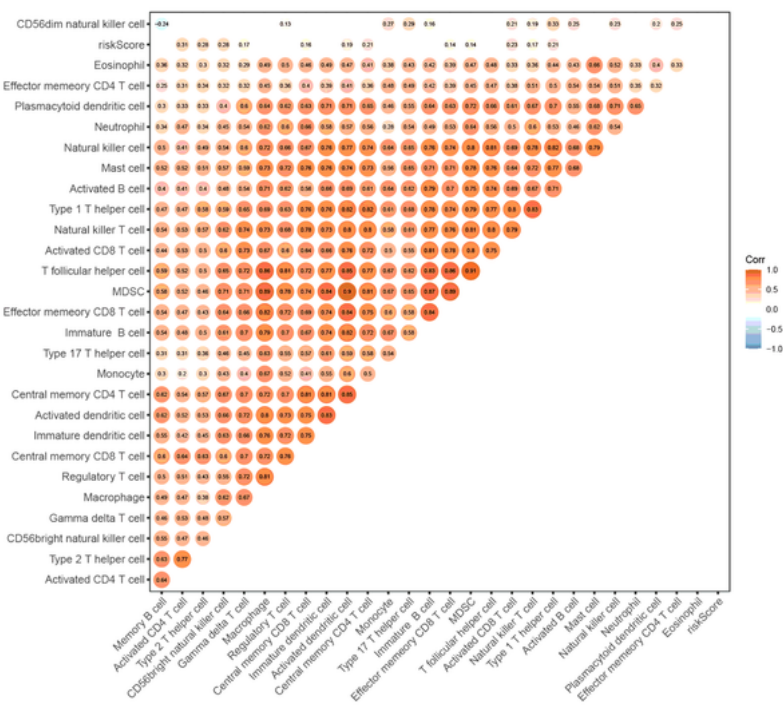

\section{Figure 5}

Correlation of the IncRNA-based Riskscore with immune infiltrates. A. The correlation coefficients of the Riskscore with Macrophages M0, Macrophages M1 and T cells CD4 memory resting were $0.27,0.32,0.25(P<0.05)$, respectively. The correlation coefficients were $R=-0.24(P<0.05)$ for Mast cells activated and $\mathrm{R}=-0.38(\mathrm{P}<0.05)$ for Monocytes, respectively. B. The correlation coefficients between the Riskscores of Macrophages M1, Plasma cells, and T cells follicular helper were $0.23,0.25$, and- 0.27 , respectively $(P<0.05)$. The correlation between ARIncRNA riskscore and 28 immune cell infiltration using single-sample geneset enrichment analysis (SsGSEA) to training set (C) and validation set (D).
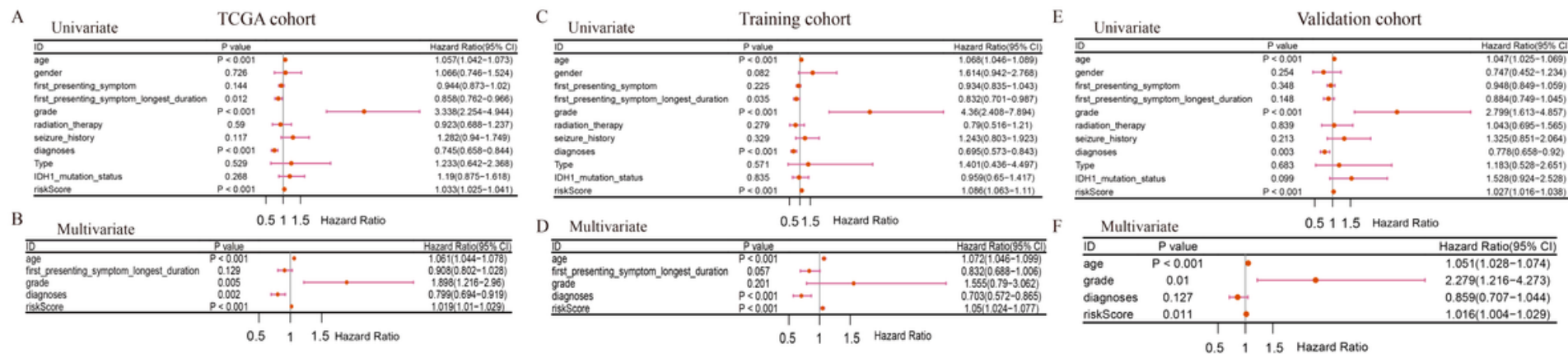

\section{Figure 6}

Verification of the IncRNA signature-based Riskscore as an independent prognostic factor. A,C,E. Univariate Cox regression analysis showed that age, grade, diagnoses and IncRNA signature-based Riskscore were significant factors of prognostic value. B, D, F. In multivariate Cox regression model, only age and IncRNA signature-based Riskscore were associated with OS, suggesting that the autophagy-related IncRNA signature is an independent prognostic biomarker for survival in LGG patients. 
A
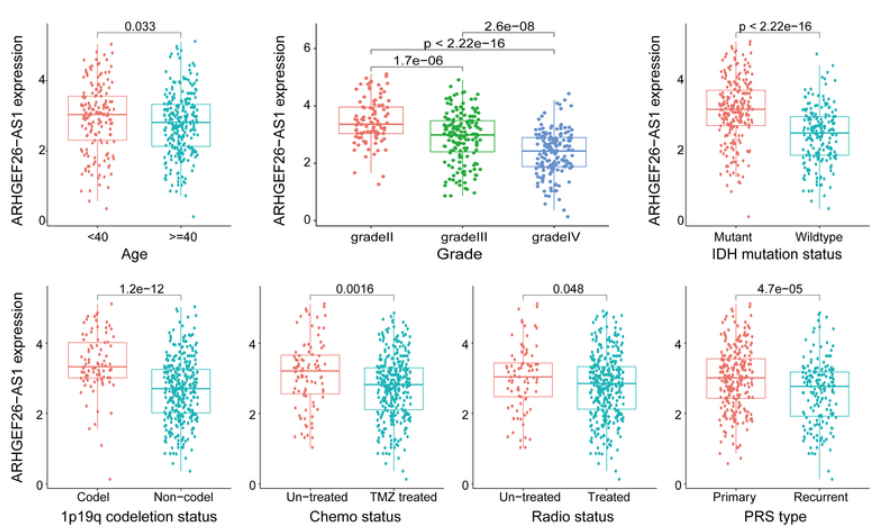

B
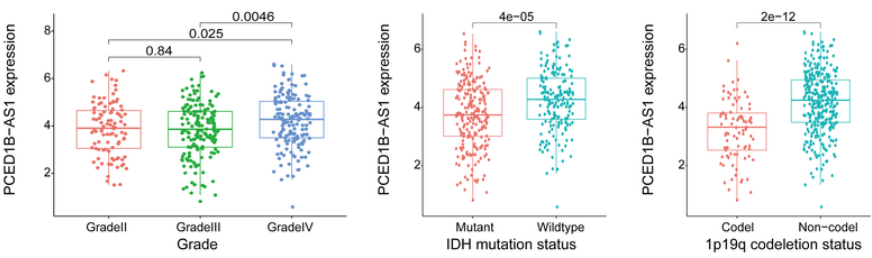

C
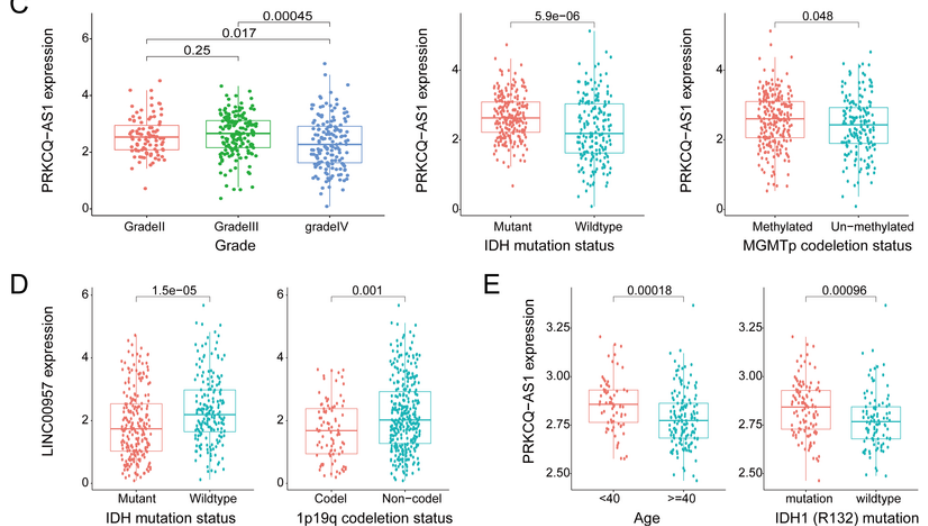

$\mathrm{E}$

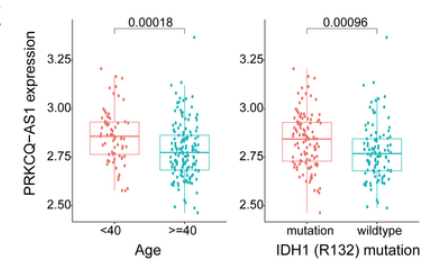

Figure 7

Assessment of ARIncRNA partial gene performance using two external independent datasets. A. Box plots of ARHGEF26-AS1 gene expression levels in patients with different age ( $<40$ and $>=40$ years), tumor grade, IDH1 mutation status, $1 \mathrm{p} 19 \mathrm{q}$ co-deletion status, chemotherapy status, radiotherapy status and PRS type. B. Box plots of PCED1B-AS1 expression in CGGA mRNA-seq693 dataset in patients with different tumor grades, IDH1 mutation status and 1p19q codeletion status status. C. Box plots of PRKCQ-AS1 expression in CGGA mRNA-seq693 dataset in patients with different tumor grades, IDH1 mutation status and MGMTp co-deletion status. D. Box plots of LINC00957 gene expression levels in patients with IDH1 mutation status and 1p19q co-deletion status in the CGGA mRNA-seq693 dataset. E. Box plots of PRKCQ-AS1 gene expression levels in patients of different ages and IDH1 (R132) mutation status. 
A
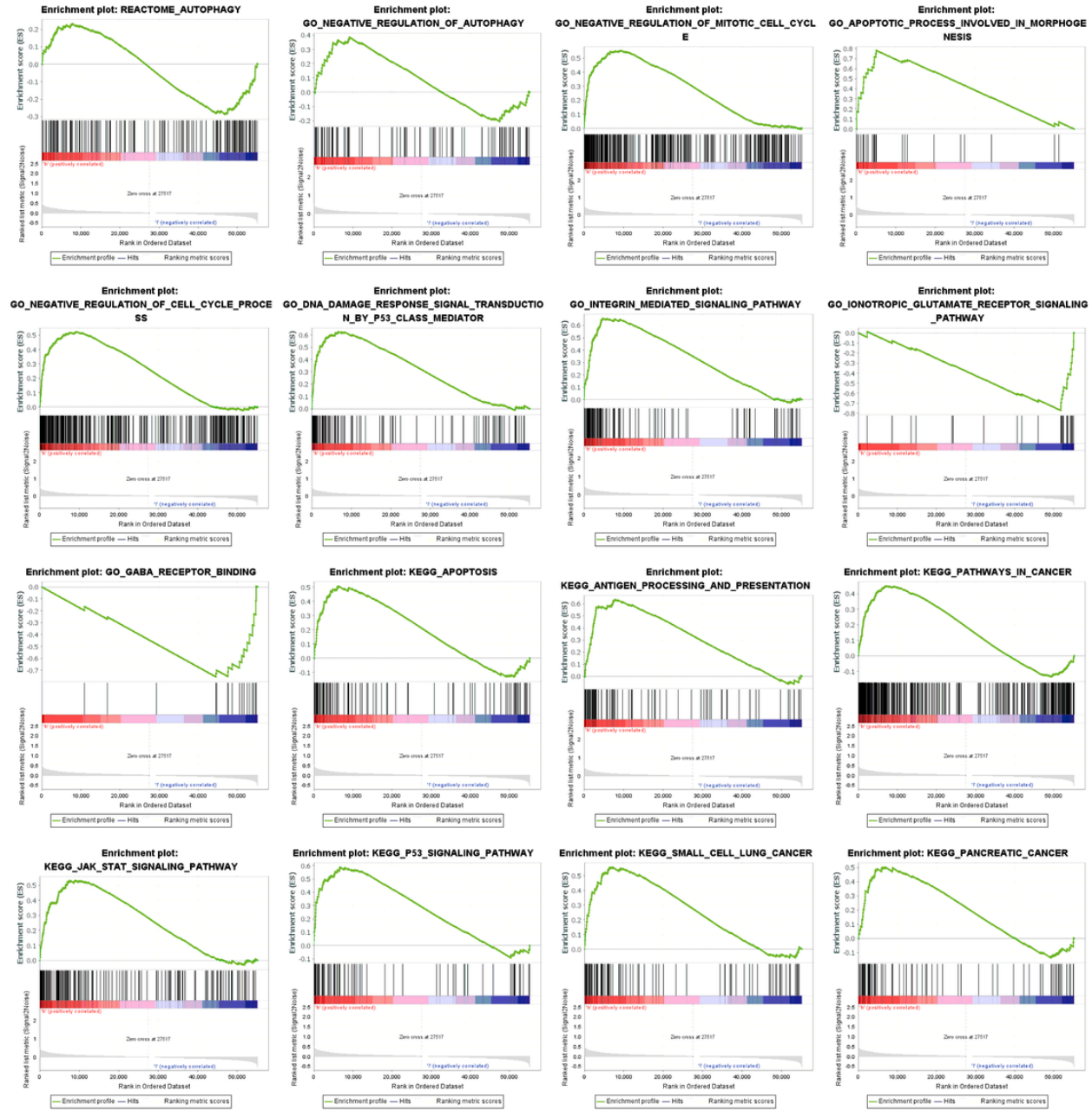

Figure 8

A. GSEA analysis demonstrates that autophagy-related phenotypes are heavily enriched in both high- and low-risk patients.

\section{Supplementary Files}

This is a list of supplementary files associated with this preprint. Click to download.

- FigureS1.tif

- Figures2.tif

- FigureS3.tif

- Tables1.xls 